\title{
Spatial localization of warmth
}

\author{
RICHARD H. TAUS, JOSEPH C. STEVENS, and LAWRENCE E. MARKS \\ John B. Pierce Foundation Laboratory and Yale University, New Haven, Connecticut 06519
}

\begin{abstract}
This study explored the ability to localize warmth sensations produced by radiant stimulation of the forearm. The subject's task was to state on which side of a tactile reference line warmth was perceived. Percentage correct improved with increasing intensity of the radiation, as well as with increasing distance from the reference line. There appears to be an inverse relation between the ability to make spatial judgments and the degree of spatial summation. At low levels of stimulation, intensity and area both contribute heavily to the magnitude of a warmth sensation and spatial localization is poor; but with increasing level, area contributes less and less to sensation and localization is better.
\end{abstract}

The warmth sense is notable for its generous spatial summation and poor spatial resolution. In the region of the absolute threshold, intensity and areal extent of stimulation contribute about equally to warmth sensation; but recent studies (Marks \& Stevens, 1973; Stevens \& Marks, 1971) have shown that as stimulus intensity progressively increases, summation decreases in that the relative contribution of area progressively diminishes. The purpose of the present study was to learn whether the decline in spatial summation might be accompanied by an increase in the capacity of the warmth sense to mediate spatial information. The particular spatial task chosen for study was the ability to localize warmth sensations caused by radiant stimulation.

What investigations there have been of the capacity of the warmth sense to mediate spatial information (e.g., size, shape, location of the stimulus) all seem to testify to its mediocrity compared with touch and vision. There is also an occasional suggestion that the capacity for spatial analysis might depend on intensity.

To begin with, Dimmick (1915) studied localization of both radiant heat and tactile pressure on the volar surface of the forearm and found the average error of localization to be far greater with heat. Pritchard (1931), working on the dorsal surface of the hand, found the average error for warmth localization to be approximately twice as great as that for touch. Neither study systematically examined different levels of irradiance. Pritchard did use several tactile intensities (different von Frey hairs) in his study of tactile localization; he concluded that the ability to localize tactile stimuli does not depend on intensity.

Stone (1937) explored form and pattern perception in the cutaneous senses, finding modest ability with passive touch but virtually none with radiant warmth.

Piéron (1919) determined two-point limens for drops of water on the skin and found that spatial

This study was supported by United States Public Health Service Grant ES-00354. Send reprint requests to Joseph C. Stevens. John B. Pierce Foundation Laboratory, 290 Congress Avenue, New Haven, Connecticut 06519. acuity was improved by increasing the displacement of water temperature, hot or cold, from neutral skin temperature. More recently, Cain (1973) examined two-tield discrimination of radiant warmth on the back (thereby avoiding tactile stimulation), and found it to be very poor indeed, although it appeared to improve with irradiance level. Cain also found that subjects even frequently confused ventral and dorsal stimulation of the torso, but made no such errors localizing very light touch. Garcia (1950) found that subjects frequently confused stimulation of the right and left sides of the forehead.

Stevens and Marks (1971) asked subjects to estimate the areal extent of irradiation of the back; the estimates obtained were very poor but more accurate at high than at low intensity.

The present experiment asked subjects to state on which side of a tactile reference line radiant warmth was perceived. The results showed that the percentage correct response improves both with increasing stimulus intensity and with increasing distance from the reference.

\section{METHOD}

\section{Apparatus and Subjects}

Sessions were conducted in a large room whose air temperature was approximately $22^{\circ} \mathrm{C}$. Warmth was produced by two projectors. each equipped with a $1,000-\mathrm{W}$ projector bulb. One projector was mounted on each side of a split platform. A knob attached to the platform permitted prompt and accurate adjustment of the distance between the projectors (Figure 1).

A blackened aluminum mask on the platform in front of each projector limited the radiant stimulation to a square tield with sides of $4.0 \mathrm{~cm}$. A hand-operated aluminum shutter was used to control the exposure time, which was always 3 sec. Intensity was controlled by varying the voltage across the lamps. A Hardy radiometer (Hardy, Wolff. \& Goodell, 1952) was used to measure irradiance, which was uniform $\pm 4 \%$ over the field. Three men served as subjects, each for 101 -h sessions.

\section{Procedure}

First. the volar surface of the right forearm was blackened with India ink to give complete absorption of the incident radiation (Hardy et al., 1952). The subject was then seated with his forearm resting in front of the projectors. Before each trial, the experimenter touched the middle (dotted line. Figure 1) of the 


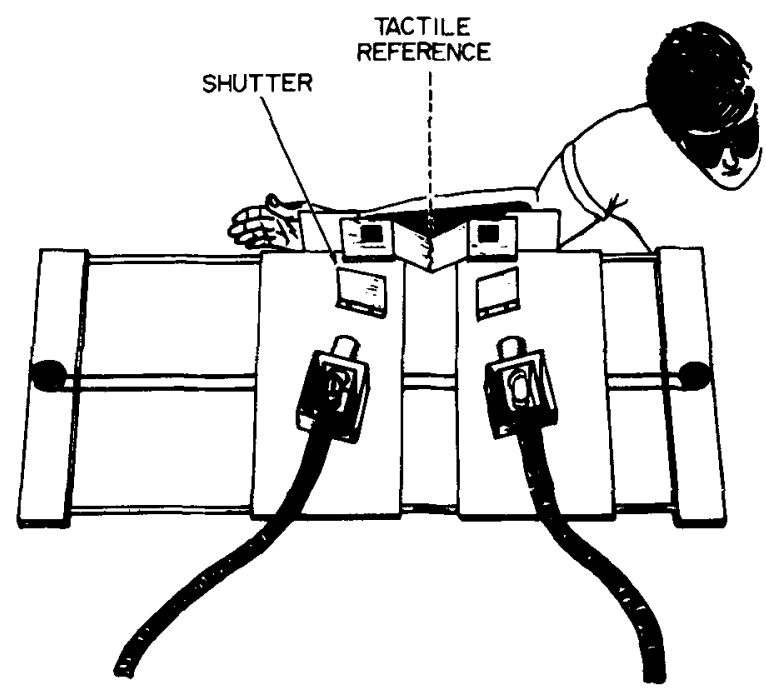

Figure 1. Schema of apparatus showing the two heat projectors, shutters, and masks.

subject's forearm with a plastic straightedge. This served as a reference line for the subject. whose task it was to state whether the subsequent thermal stimulus was presented to the proximal ("clbow") or distal ("wrist") half of the forearm. When a stimulus was not perceived, as occasionally occurred with the lowest intensity, the same stimulus was presented again later. Those trials on which the subject reported no sensation were not counted in the data analysis. Three distances from the reference to the center of the field were used, $2.5,4,5.5 \mathrm{~cm}$; each stimulus location was stimulated at four different irradiance levels, 68, 138, 224, and $398 \mathrm{~mW} / \mathrm{cm}^{2}$. A session comprised 10 presentations of each distance and intensity combination: 5 presentations on the distal half and 5 on the proximal. A complete session, therefore, included 120 perceived presentations. Order of presentation was random. Rate of stimulus presentation was approximately one every $30 \mathrm{sec}$.

The subjects were blindfolded in order to control visual cues; noise produced by the blowers that cooled the lamps helped to mask potential auditory clues (i.e., sound of shutters). The subjects were never told whether their responses were correct, and it would therefore have been difficult for them to correlate any nonthermal stimulus with a particular response.

\section{RESULTS AND DISCUSSION}

The measure of spatial accuracy used here is simply percentage of correct responses. In the present task, with only two alternatives, a score of $50 \%$ correct can be attributed to chance (no discrimination). The scores ranged from $58.7 \%$ for the lowest intensity at the smallest distance from the reference to $94.3 \%$ for the highest intensity at the greatest distance from the reference. Although these are averages for the three subjects, all three behaved in about the same way. The scores for equal displacements (e.g., $2.5 \mathrm{~cm}$ distal and $2.5 \mathrm{~cm}$ proximal to reference) were also averaged. although there was a slight tendency on the part of all three subjects to localize more frequently on the proximal side.

As Figure 2 shows, the percentage correct response improved both with increased distance and with higher intensity. As an example, $66.3 \%$ accuracy was achieved at al distance of $2.5 \mathrm{~cm}$ and an intensity of
$138 \mathrm{~mW} / \mathrm{cm}^{2}$. This score could be raised the same amount either by increasing the intensity to $398 \mathrm{~mW} / \mathrm{cm}^{2}$ or by increasing the distance to $5.5 \mathrm{~cm}$. In this particular tradeoff, an increase of $188 \%$ in intensity is equivalent to an increase of $120 \%$ in distance; it is representative of the data in that distance was somewhat effective, but not much more so than intensity, in improving the score. That is, although the effects are of the same order of magnitude, a simple lever law does not apply.

One of the subjects also served in a small study of tactile localization under conditions similar to those used here for warmth localization. Again, the subject was blindfolded and touched with a straightedge before each presentation, but von Frey hairs were used as stimuli at distances from the reference of 2.5 , 4.0 , and $5.5 \mathrm{~cm}$. Accuracy of $99 \%$ was achieved over 200 trials, even though the hair used was just barely perceptible. With a stiffer hair, no errors at all occurred in 100 trials. The tactile stimuli were evenly divided between proximal and distal halves, and nearly evenly among the three distances. Thus the tactile sense displays far superior spatial localization and, as reported by Pritchard (1931), reveals no obvious dependence on intensity.

The outcome of the experiment adds to earlier evidence that the capacity of the warmth sense to mediate spatial information improves with increasing level of stimulation, while at the same time the degree of spatial summation declines. It may be that the extensive convergence of neural signals from large areas of the skin, implied by the generous spatial summation, is what limits spatial accuracy at low levels; if this is so, then it is reasonable to suppose that the decline in spatial summation at higher levels permits the skin to mediate spatial information more accurately.

Why does summation decline and spatial accuracy

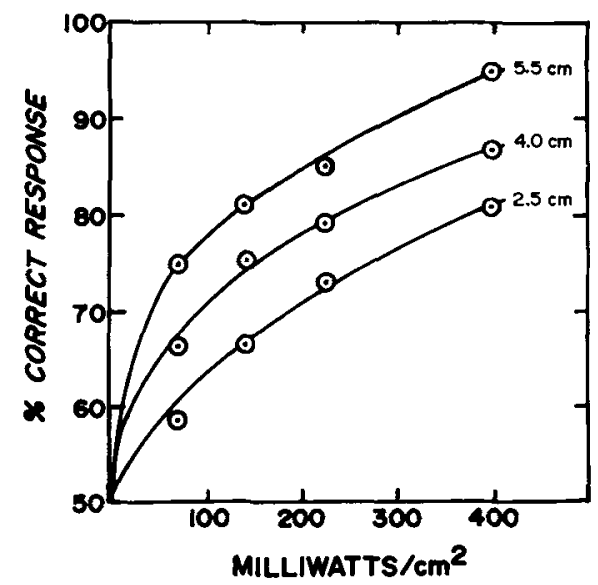

Figure 2. Percentage of correct localizations of radiant warmth on the forearm as a function of stimulus intensity. The parameter is the distance from the center of the stimulus field to a tactile reference line. 
improve with level? Until the appropriate neurophysiological measurements are made, we can only speculate. Stevens and Marks (1971) suggested two possible mechanisms that could account for the decline in summation; both could also account for the improvement in spatial accuracy. One explanation postulates the existence of two receptor populations: a population that predominates at low intensities and shows extensive summation, but poor acuity; another that predominates at high intensities and shows less summation, but greater acuity. An alternative explanation invokes neural inhibition. Psychophysical evidence from vision and hearing suggests that inhibition increases as intensity increases (Stevens, 1966). Increasing inhibition could serve in the warmth sense both to counteract summation and to sharpen spatial acuity.

Whatever the mechanism, the shifts in summation and acuity have biological significance: when stimulus intensity is low, summation predominates. The sensory response tends to depend on the total heat input, i.e.. the irradiance integrated over area. This integrated quantity is significant as a stimulus for physiological and behavioral processes of body temperature regulation. But, with intense heat, improved localization is obviously needed to avoid local damage by burning.

\section{REFERENCES}

Cans, W. S. Spatial discrimination of cutaneous warmth. American Jourmal of Psichology. 1973, 86. 169-181.

Dimmick. F. L. On the iocalization of pure warmth sensations. Americun Jourmal of Psichology. 1915. 26. 142-150

Garcia. J. P. A study of the neural mechanisms involved in the spatial summation of heat sensation. Unpublished Master's thesis. Connell University, 1950.

Hardy, J D., Wolff. H. G.. \& Goodell. H. Path se'hsations and rections. Baltimore: Williams and Wilkins. 1952.

Marks. L. E., \& Stevens, J. C. Spatial summation of warmth: Influence of duration and contiguration of the stimulus. American Jourmal of Psychology. 1973, 86, 251-267.

Piéron. H. De la discrimination spatiale des sensations thermiques: Son importance porer la théorie générale de la discrimination cutanée. Comptes Rendus de la Société de Biologie, 1919, 82, 61-65.

Pritchard, E. A. B. Cutaneous tactile localization. Brain, 1931, 54, 350-371.

Stevens. J. C.. \& Marks, L. E. Spatial summation and the dynamics of warmth seusation. Perception \& Psychophysics, $1971,9,391-398$.

Stevens, S. S. Power-group transformations under glare. masking. recruitment. Jounal of the Acoustical Society of Americu, 1966, 39. 725-735.

STONE. L. J. An experimental study of form perception in the thermal senses. Psychological Record, 1937, 1. 234-337.

(Received for publication April 1, 1974: accepted October 25, 1974.) 